\title{
Rotational quantum beat lasing without inversion
}

\author{
Maria Richter, ${ }^{1, \dagger, *}$ Marianna Lytova ${ }^{2,5, \dagger}$ Felipe Morales, ${ }^{1}$ Stefan Haessler ${ }^{3}$ \\ Olga Smirnova, ${ }^{1,4}$ Michael Spanner, ${ }^{2,5}$ and Misha Ivanov ${ }^{1,6,7}$ \\ ${ }^{1}$ Max-Born-Institute, Max-Born Straße 2A, 12489 Berlin, Germany \\ ${ }^{2}$ Department of Physics, University of Ottawa, Ottawa K1N 6N5, Canada \\ ${ }^{3}$ Laboratoire d'Optique Appliquée, CNRS, École Polytechnique, ENSTA Paris, Institut Polytechnique de Paris, 181 Chemin de la Hunière et des \\ Joncherettes, 91120 Palaiseau, France \\ ${ }^{4}$ Technische Universität Berlin, Straße des 17. Juni 135, 10623 Berlin, Germany \\ ${ }^{5}$ National Research Council of Canada, 100 Sussex Drive, Ottawa K1A OR6, Canada \\ ${ }^{6}$ Department of Physics, Humboldt University, Newtonstraße 15, 12489 Berlin, Germany \\ ${ }^{7}$ Blackett Laboratory, Imperial College London, SW72AZ London, UK \\ *Corresponding author: maria.richter@mbi-berlin.de
}

Received 21 February 2020; revised 14 April 2020; accepted 17 April 2020 (Doc. ID 390665); published 27 May 2020

\begin{abstract}
In standard lasers, light amplification requires population inversion between an upper and a lower state to break the reciprocity between absorption and stimulated emission. However, in a medium prepared in a specific superposition state, quantum interference may fully suppress absorption while leaving stimulated emission intact, opening the possibility of lasing without inversion. Here we show that lasing without inversion arises naturally during propagation of intense femtosecond laser pulses in air. It is triggered by the combination of molecular ionization and molecular alignment, both unavoidable in intense light fields. The effect could enable inversionless amplification of broadband radiation in many molecular gases, opening unusual opportunities for remote sensing.
\end{abstract}

Published by The Optical Society under the terms of the Creative Commons Attribution 4.0 License. Further distribution of this work must maintain attribution to the author(s) and the published article's title, journal citation, and DOI.

https://doi.org/10.1364/OPTICA.390665

\section{INTRODUCTION}

A resonant light propagating through a medium will be absorbed by the medium in a lower state and will stimulate emission if the medium is excited. For a quantum system pumped into an excited state $|e\rangle$, with an empty state $|g\rangle$ below, population inversion between these two states leads to amplification of a probe light at the $|e\rangle \rightarrow|g\rangle$ transition frequency. For almost six decades, this process has been exploited in conventional lasers. However, three decades ago, it was shown that lasing can also occur without population inversion [1-8].

The basic idea behind lasing without inversion (LWI) [9-11] is similar to conventional lasers- to prepare a medium where emission is favored over absorption so that resonant light is amplified as it propagates in the medium. The difference lies in the medium preparation. Instead of creating population inversion between the lasing states, quantum interference is used to suppress photoabsorption while keeping photoemission intact. In the simplest scheme, two lower-lying states $\left|g_{1}\right\rangle$ and $\left|g_{2}\right\rangle$ are each coupled to a common upper state $\left|e_{1}\right\rangle$. One can prepare a coherent superposition of the two lower states such that the transition from this superposition to the upper state vanishes due to destructive interference of the transition amplitudes $\left|g_{1}\right\rangle \rightarrow\left|e_{1}\right\rangle$ and $\left|g_{2}\right\rangle \rightarrow\left|e_{1}\right\rangle$. Now any population pumped incoherently into the upper state can provide gain.
A number of different schemes for lasing without inversion have been developed $[9,10]$. Taking advantage of destructive interference of different pathways in absorption, they generally strive to maintain a specific phase relationship between the lower-lying states, which carry most of the population. Here we present a scheme that does not follow this tradition. It uses only the natural dynamics of a multilevel quantum system and requires no coherence between the excited and the lower electronic states; effectively, lasing without inversion comes "for free." We also show that this mechanism is active in the highly efficient generation of $391 \mathrm{~nm}$ radiation during propagation of intense femtosecond laser pulses in air [12-16], under standard conditions, where the process known as laser filamentation [17-19] leads to self-guiding of light. The $391 \mathrm{~nm}$ line corresponds to the transition between the two ground vibrational levels $v^{\prime \prime}, v^{\prime}=0$ of the ground electronic state $X^{2} \Sigma_{g}^{+}$(denoted as $X$ ) and the second excited state $B^{2} \Sigma_{u}^{+}$ (denoted as $B$ ) in $\mathrm{N}_{2}^{+}$.

Identifying the mechanism responsible for the amplification, commonly referred to as "air lasing," has been a long-standing puzzle [20-45]. The main difficulty in resolving this puzzle stems from the apparent lack of a general physical mechanism capable of generating population inversion between the states $X$ and $B$ for standard filamentation conditions, including the clamping of the laser intensity around $I \sim 10^{14} \mathrm{~W} / \mathrm{cm}^{2}[46-48]$. 
In the following section, we introduce the general scheme that can enable inversionless amplification of light in gas molecules, solely by excitation with a short, intense laser pulse. In its simplest form, this mechanism can be referred to as rotational quantum beat lasing without inversion. Subsequently, we examine the specific example of the amplification around the $391 \mathrm{~nm}$ line and show that this mechanism provides the key missing component of the puzzle. In the recent work of Ref. [35], a different lasing without an inversion mechanism has been proposed to cause the gain at the $391 \mathrm{~nm}$ line based on the existence of long-lived coherent polarizations coupling simultaneously the states $X, B$, and the intermediate first excited electronic state $A$ in $\mathrm{N}_{2}^{+}$. The present amplification scheme relies on rotational dynamics and does not require coherences between the electronic states of the ion, even though such coherence is inevitably created by the pump pulse driving the molecular ion after ionization of the neutral. Compared to the model in Ref. [35], we account for the light-induced vibrational and rotational dynamics of the molecule, which allows us to capture the effect of rotational quantum beat lasing without inversion.

\section{ROTATIONAL QUANTUM BEAT LASING}

The mechanism of rotational quantum beat lasing is illustrated in Fig. 1, and it can be easily understood in the time domain. When an intense femtosecond laser pulse interacts with a diatomic molecule, or its ion, it induces molecular alignment [49,50]. This alignment revives periodically after the pulse is gone [Fig. 1(a)]. The revival dynamics demonstrate a sequence of alignment and anti-alignment with the revival period controlled by the rotational constant $B^{0}$. Figure 1 (a) shows the evolution of the characteristic alignment measure, $\left\langle\cos ^{2} \theta\right\rangle(t)$, for a nitrogen molecule at room temperature $(298 \mathrm{~K})$, after interacting with a $23 \mathrm{fs} F W H M$, $800 \mathrm{~nm}$ pump pulse with a peak intensity of $10^{14} \mathrm{~W} / \mathrm{cm}^{2}$ (see Supplement 1). Its minima correspond to anti-alignment and its maxima to maximum alignment with the direction of the linearly polarized aligning laser field.

Suppose now that similar rotational dynamics are induced in two electronic (vibronic) states, $|1\rangle$ and $|2\rangle$, with a dipole-allowed electronic transition between them. In diatomics, electronic transitions can be either parallel or perpendicular to the molecular axis; for definiteness, let this transition be parallel. Its probability is then proportional to $\cos ^{2} \theta$, where $\theta$ is the angle between the molecular axis and the laser electric field inducing the transition. For a molecule rotating in the lower vibronic state, the probability of parallel absorption $|1\rangle \rightarrow|2\rangle$ thus depends on $\left\langle\cos ^{2} \theta\right\rangle_{1}(t)$ averaged over the rotational dynamics in state $|1\rangle$, which is controlled by its rotational constant $B_{1}^{0}$. Conversely, for a molecule rotating in the upper vibronic state $|2\rangle$, the emission probability $|2\rangle \rightarrow|1\rangle$ depends on $\left\langle\cos ^{2} \theta\right\rangle_{2}(t)$, with its time dependence controlled by $B_{2}^{0}$.

Opportunities for inversionless amplification of a short probe pulse, polarized parallel to the pump and delayed by $\tau$, arise when the $|1\rangle$ state molecules are anti-aligned, so that $\left\langle\cos ^{2} \theta\right\rangle_{1}(\tau)$ is minimum and the parallel $|1\rangle \rightarrow|2\rangle$ absorption is suppressed. This opportunity is further enhanced if the $|2\rangle$ state molecules are aligned at this time, so that $\left\langle\cos ^{2} \theta\right\rangle_{2}(\tau)$ is maximum and the parallel $|2\rangle \rightarrow|1\rangle$ emission is enhanced. This possibility to use molecular alignment for inversionless amplification was previously pointed out by Popov and Slabko [51]. The converse is true if the $|1\rangle$ state molecules are aligned when the probe pulse arrives, while the $|2\rangle$ state molecules are anti-aligned, enhancing absorption. As long as $B_{1}^{0}$ and $B_{2}^{0}$ are different, the two rotations will go out of sync, arriving at the point where the lower state is anti-aligned and the upper is still aligned and vice versa [Fig. 1(b)]. Overall, temporal windows of gain will be followed by windows of loss, leading to the rotational quantum beats in the time-resolved gain-loss of the short probe pulse.
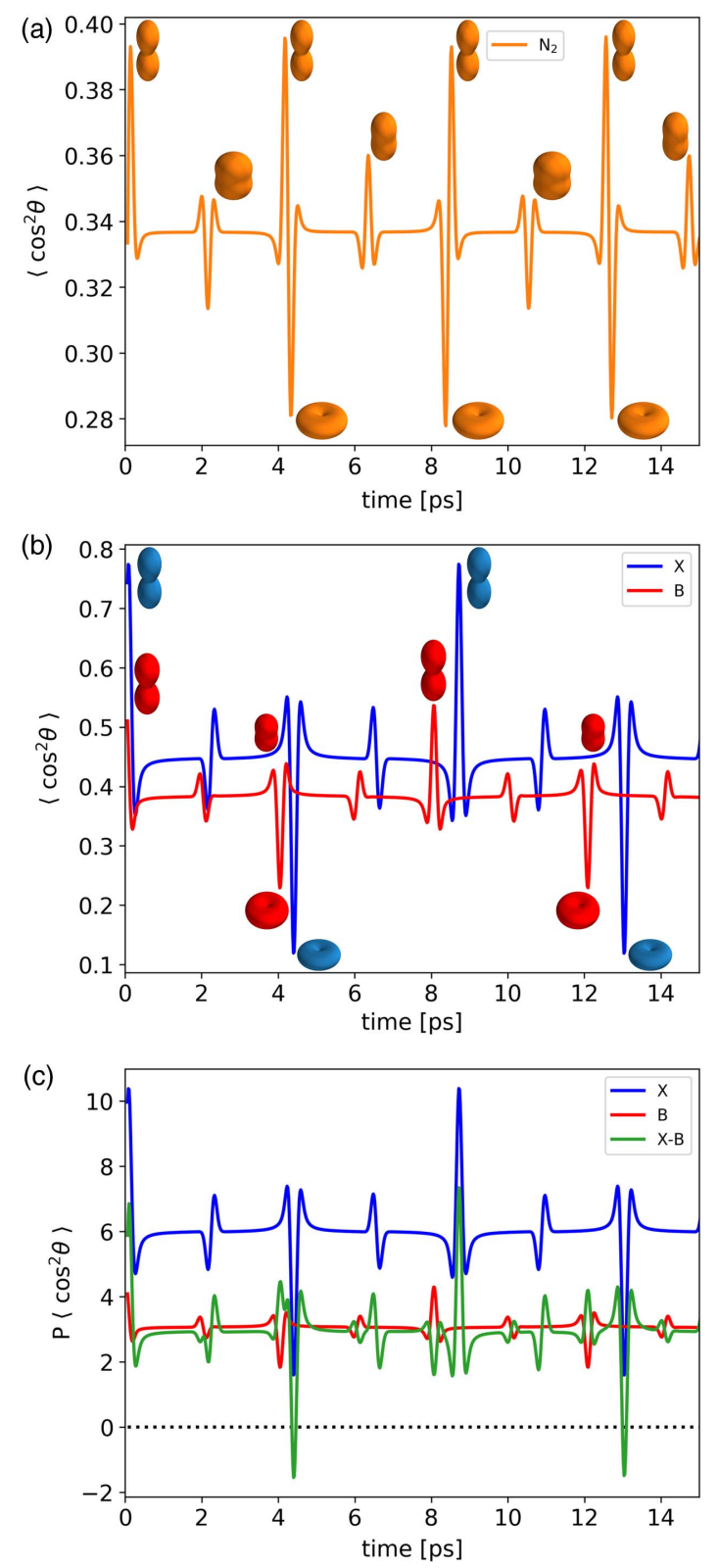

Fig. 1. Schematic of a rotational quantum beat laser without inversion in the time domain using the example of $\mathrm{N}_{2}^{+}$. Calculations are performed for a $23 \mathrm{fs}, 800 \mathrm{~nm}, 10^{14} \mathrm{~W} / \mathrm{cm}^{2}$ pump pulse and room temperature $(298 \mathrm{~K})$; see text and Supplement 1 for details. The 3D shapes in panels (a),(b) sketch the angular distributions of the rotating molecular ensembles. (a) Field-free molecular alignment of $\mathrm{N}_{2}$ at $298 \mathrm{~K}$ induced by the intense femtosecond pump pulse. (b) Field-free rotational dynamics in the two vibronic states $\left|X, v^{\prime \prime}=0\right\rangle$ and $\left|B, v^{\prime}=0\right\rangle$ of $\mathrm{N}_{2}^{+}$induced by the same pump. (c) $P_{X}\left\langle\cos ^{2} \theta\right\rangle_{X}(\tau)$ (blue), $P_{B}\left\langle\cos ^{2} \theta\right\rangle_{B}(\tau)$ (red), and $P_{X}\left\langle\cos ^{2} \theta\right\rangle_{X}(\tau)-P_{B}\left\langle\cos ^{2} \theta\right\rangle_{B}(\tau)$ (green). Gain windows (at $\tau_{1} \simeq 4.4 \mathrm{ps}$ and $\tau_{2} \simeq 13.0 \mathrm{ps}$ ) without population inversion at a parallel transition between the two vibronic states are enabled by the different angular distribution geometries of the rotating molecular ensembles in the upper and lower state; no coherence between the two rotational manifolds is required. 
Figures 1(b) and 1(c) give the specific example. Figure 1(b) shows the rotational dynamics in the two ground vibrational levels $v^{\prime \prime}, v^{\prime}=0$ of the electronic states $X$ and $B$ in $\mathrm{N}_{2}^{+}$, induced by the same pump pulse interacting with $\mathrm{N}_{2}$ [Fig. 1(a)]. The short pump pulse impulsively aligns the neutral $\mathrm{N}_{2}$ molecules, generating the rotational dynamics in Fig. 1(a). It also ionizes some of the $\mathrm{N}_{2}$ molecules, producing molecular ions predominantly in the ground state $X$ but also in the excited $A\left(A^{2} \Pi_{u}\right)$ and $B$ states, and it continues to align them. After the end of the pulse, the ions continue to rotate, reaching maximum alignment at $t \simeq 90 \mathrm{fs}$ for the $X$ and $B$ states [see Fig. 1(b)], followed by periodic revivals of alignment and anti-alignment. They correspond to maxima and minima in the rotational ensemble-averaged $\left\langle\cos ^{2} \theta\right\rangle_{X, B}(t)$, where subscripts denote the ionic states (see Supplement 1). These revivals are different due to slight differences in the rotational constants $B_{X}^{0}$ and $B_{B}^{0}$.

Frequency-integrated absorption at the parallel $\left|X, v^{\prime \prime}=0\right\rangle \rightarrow$ $\left|B, v^{\prime}=0\right\rangle$ transition behaves as $W_{\text {abs }}(\tau) \propto P_{X}\left\langle\cos ^{2} \theta\right\rangle_{X}(\tau)$, where $P_{X}$ is the $\left|X, v^{\prime \prime}=0\right\rangle$-state population (see Supplement 1). In emission, $W_{\mathrm{e}}(\tau) \propto P_{B}\left\langle\cos ^{2} \theta\right\rangle_{B}(\tau)$. Gain windows open when $P_{B}\left\langle\cos ^{2} \theta\right\rangle_{B}(\tau)>P_{X}\left\langle\cos ^{2} \theta\right\rangle_{X}(\tau)$; see Fig. 1(c). The populations of the $X$ and $B$ states, $P_{X, B}$, determine the length and the strength of the gain windows; however, overall electronic population inversion is not needed [Fig. 1(c)]. The comprehensive quantitative analysis presented below shows that this naturally arising amplification mechanism does indeed lead to gain at the rotational frequency band around $391 \mathrm{~nm}$ during filamentation of intense femtosecond laser pulses in air or $\mathrm{N}_{2}$ gas, including the intensity regime $I \lesssim 10^{14} \mathrm{~W} / \mathrm{cm}^{2}$ pertinent to laser filamentation.

\section{THEORETICAL FRAMEWORK}

Most experiments studying the amplification effect around the $391 \mathrm{~nm}$ line in $\mathrm{N}_{2}^{+}$are done in a pump-probe scenario [14,2022,24,26,27,30-32,34-39,41-44]: an intense pump pulse prepares the gas, and the transient absorption (or gain) of a delayed probe pulse is measured. The theoretical results presented below focus on this scenario, with the pump pulse carried at $800 \mathrm{~nm}$. Details of our simulations are given in Supplement 1, and here we outline key issues requiring particular attention when addressing this rather challenging problem quantitatively.

Our theoretical description accounts for laser-induced alignment of the neutral $\mathrm{N}_{2}$ molecule, its alignment-dependent strong-field ionization into the laser-dressed states of the molecular ion, and full laser-induced electronic, vibrational, and rotational dynamics in the ion involving the $X, A, B$ states. Our ab initio simulations of strong-field ionization use the method of Ref. [52] and allow us to evaluate the excitation of the different ionic states induced by the recollision of the photoelectron with the parent ion in the $X$ state $[23,24,33-35,40]$. The role of recollision is gauged by absorbing the electron wave packet before it is turned around towards the parent ion. Eliminating the recollision reduces the population of the $B$ state by about $1 \%$ compared to the case when the recollison is included. Thus, the recollision can be neglected and the photoelectron can be integrated out. Here, one must account for the electron-ion entanglement. Since the $X$ and $B$ states have opposite parity, the photoelectron wave packets correlated to them will also carry opposite parity, remaining orthogonal to each other. This eliminates the coherence between the $X$ and $B$ states that could have been produced during strong-field ionization.
Regarding optical $X \rightarrow B$ excitation by an $800 \mathrm{~nm}$ pump, the $\left|X, v^{\prime \prime}=0\right\rangle \rightarrow\left|B, v^{\prime}=0\right\rangle$ transition corresponds to absorption of two photons, which is parity forbidden; noticeable excitation is only generated at very high intensities $I \gtrsim 4 \times 10^{14} \mathrm{~W} / \mathrm{cm}^{2}$ (the domain of several recent pump-probe experiments but not relevant for standard laser filamentation conditions) when the system is strongly distorted and higher-order multiphoton transitions can occur. In this context, note that strong-field ionization populates not field-free $[25,41,42,45,53]$ but already polarized (dressed by the field) ionic $X, A$, and $B$ states. Indeed, optical tunneling results from polarization of the many-body wave function of the neutral as one of the polarized electrons leaks through the potential barrier, leaving other electrons polarized. Comparison to ab initio simulations [52] shows that initializing population in the field-free ionic states at the peaks of the instantaneous electric field, where strongfield ionization takes place, generates spurious excitations of the $A$ and $B$ states due to effectively abrupt turn-on of the laser-ion interaction.

Moreover, the initial thermal rotational distribution must be included. Each rotational state in the initial thermal distribution gives rise to a rotational wave packet induced by the pump. Each wave packet leads to coherent effects in emission and absorption; these contributions are added incoherently with the corresponding weights.

With these aspects accounted for, our calculations reveal a robust physical mechanism leading to gain at the $391 \mathrm{~nm}$ line. In contrast to Ref. [35], it is based on the mechanism described in Fig. 1; it requires neither parametric coupling between $B$ and $A$ through quasi-resonant coupling of both states to $X$ via the pump and the probe nor population inversion between $B$ and $A$. Efficient population depletion from $X$ into $A$ via resonant coupling after ionization, driven by the pump pulse, is favorable for the balance between gain and loss; however, the rotational quantum beat lasing mechanism does not critically depend on the one-photon resonance. In our amplification scenario, the probe pulse follows the pump pulse without overlapping in time, so that the mechanism described in Ref. [35] is inoperative.

\section{RESULTS AND DISCUSSION}

Figure 2(a) shows the frequency-resolved transient absorption as a function of the pump-probe time-delay for a weak $20 \mathrm{fs}$ Gaussian probe pulse with a central wavelength of $391 \mathrm{~nm}$. Each vertical line shows the frequency-resolved absorption for a particular pumpprobe time delay, integrated over the full duration of the probe pulse. The horizontal white line around $\tilde{v} \simeq 25575 \mathrm{~cm}^{-1}$ marks the energy spacing $\tilde{v}_{00}$ between the two ground rovibrational levels of the electronic states $X$ and $B$. The spectral lines above this energy are associated with the higher-energy branch ( $\mathrm{R}$ branch). Those below $25575 \mathrm{~cm}^{-1}$ to the lower-energy branch (P branch).

Time-dependent gain (red) emerges for virtually all pumpprobe time delays and many transition frequencies, especially those associated with the lower-energy P branch. Most notably, for those time delays at which the $X$ state molecules are anti-aligned with the probe polarization direction, e.g., around $\tau \simeq 4.4$ ps [cf. Fig. 1(b)], amplification occurs across the whole range of frequencies.

The details of the time- and frequency-dependent transient absorption are readily understood considering the rotational quantum beat lasing mechanism in the frequency domain. The short, intense pump pulse aligning and ionizing the $\mathrm{N}_{2}$ molecules 

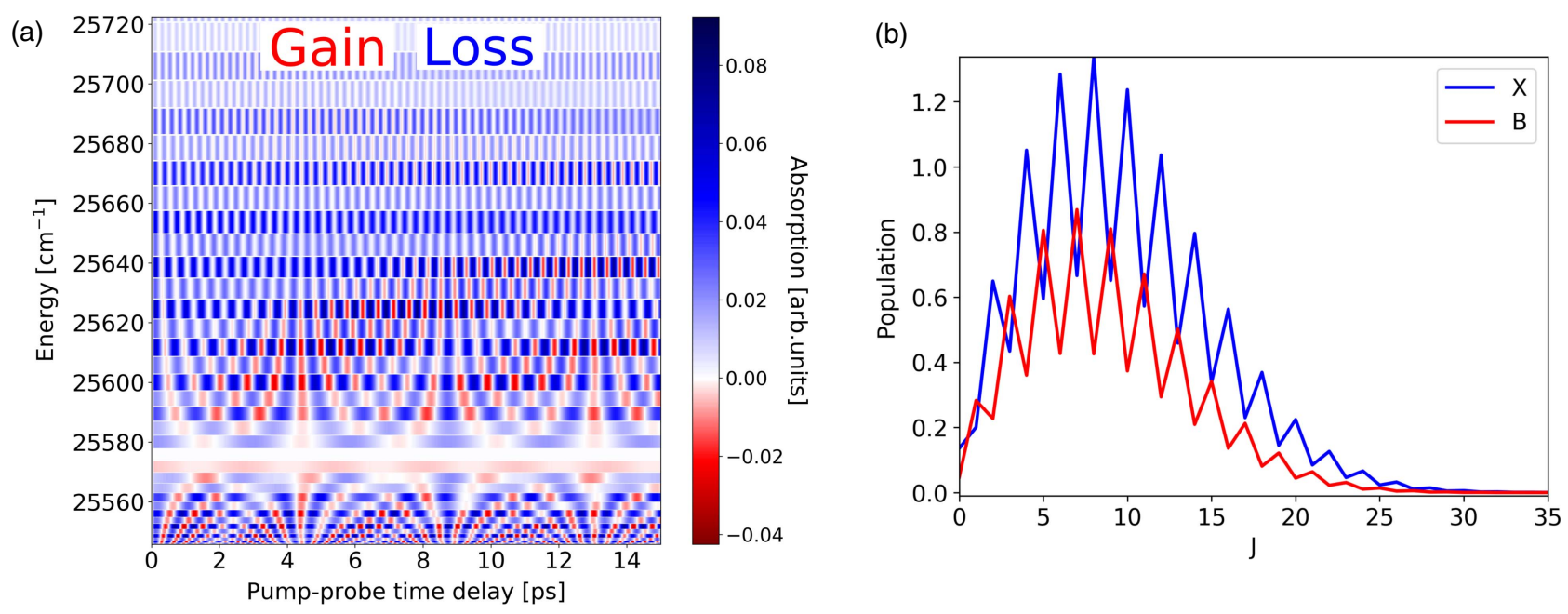

(c)

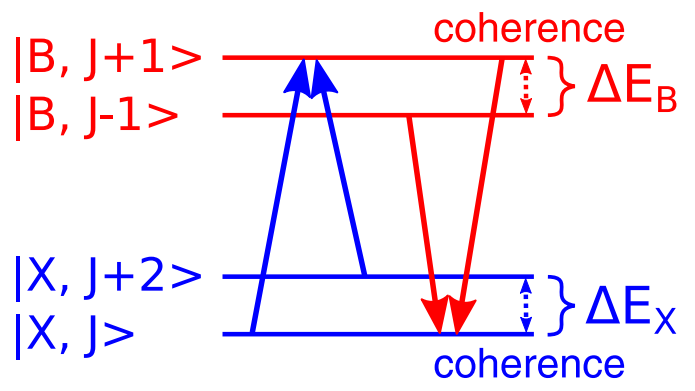

(d)

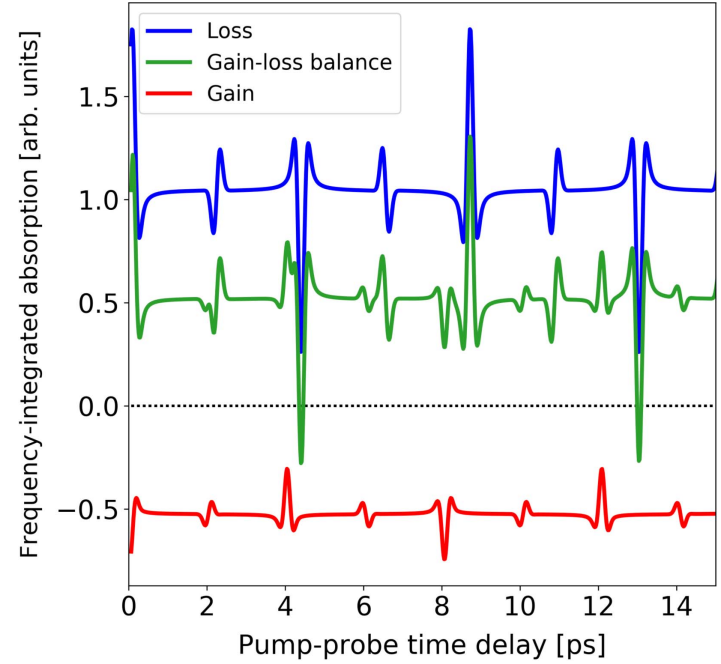

Fig. 2. Rotational quantum beat lasing without inversion in the frequency domain using the example of $\mathrm{N}_{2}^{+}$. Calculations are performed for the same pump pulse and temperature as in Fig. 1 and a $20 \mathrm{fs}, 391 \mathrm{~nm}, 10^{11} \mathrm{~W} / \mathrm{cm}^{2}$ probe pulse; see text and Supplement 1 for details. (a) Full simulation of the frequency-resolved gain (red) and loss (blue) of the weak probe interacting with the pumped system as a function of pump-probe delay. (b) Rotational distributions in the ground vibrational levels of the $X$ and $B$ states of $\mathrm{N}_{2}^{+}$formed at the end of the interaction with the intense femtosecond pump pulse. (c) Four-level system controlling absorption and emission at the $\left|X, v^{\prime \prime}=0, J\right\rangle \rightarrow\left|B, v^{\prime}=0, J+1\right\rangle$ transition. (d) Absorption (blue) and emission (negative absorption) (red) integrated over all frequencies follow the alignment dynamics in Fig. 1(b). The total gain-loss balance integrated over all frequencies (green) follows the pattern of $P_{X}\left\langle\cos ^{2} \theta\right\rangle_{X}-P_{B}\left\langle\cos ^{2} \theta\right\rangle_{B}$ in Fig. 1(c), showing the same gain windows for the inversionless medium $\left(P_{X}>P_{B}\right)$.

generates broad rotational distributions in the $X$ and $B$ states of $\mathrm{N}_{2}^{+}$, with coherent excitation of many adjacent rotational states with similar amplitudes in each electronic state. Figure 2(b) shows the rotational distributions in $\left|X, v^{\prime \prime}=0\right\rangle$ and $\left|B, v^{\prime}=0\right\rangle$ for the case of the $800 \mathrm{~nm}, 23 \mathrm{fs}$ FWHM pump pulse with an intensity of $I=10^{14} \mathrm{~W} / \mathrm{cm}^{2}$ and the $\mathrm{N}_{2}$ molecules initially at room temperature $(298 \mathrm{~K})$.

Consider now frequency-resolved transient absorption of a short probe pulse at the dipole-allowed transition $|X, J\rangle \rightarrow|B, J+1\rangle$ (with the vibrational quantum number equal to zero in both cases); see Fig. 2(c). For each initial rotational state of the neutral, rotational coherence generated in the $X$ state of the ion means that the absorption $|X, J\rangle \rightarrow|B, J+1\rangle$, stimulated by a broadband probe, is inevitably accompanied by the absorption $|X, J+2\rangle \rightarrow|B, J+1\rangle$ stimulated by the same probe; see Fig. 2(c). Their interference is governed by the relative phase between the two lower states $\quad \phi_{X}(J+2, J)(t)=\Delta E_{X}(J+2, J) t+\phi_{X}^{0}(J, J+2)$.
Here $\Delta E_{X}(J+2, J)=B_{X}^{0}[(J+2)(J+3)-J(J+1)]$ is the distance between the two rotational states in $X$, and $\phi_{X}^{0}$ is their relative phase at the start of the field-free evolution when the pump is over. Destructive interference between the $|X, J\rangle \rightarrow|B, J+1\rangle$ and $|X, J+2\rangle \rightarrow|B, J+1\rangle$ transitions occurs when $\phi_{X}(J+2, J)(t)=(2 N \pm 1) \pi$ ( $N$ is an integer), resulting in suppression of absorption of a short probe pulse arriving at this moment. Conversely, constructive interference enhances absorption when $\phi_{X}(J+2, J)(t)=2 N \pi$. This leads to the quantum beat in the time-dependent absorption rate of the short probe pulse with frequency $\Delta E_{X}(J+2, J)$.

Emission at the same frequency, $|B, J+1\rangle \rightarrow|X, J\rangle$, is inevitably accompanied by the transition $|B, J-1\rangle \rightarrow|X, J\rangle$; see Fig. 2(c). Their interference is governed by the relative phase $\phi_{B}(J+1, J-1)(t)=\Delta E_{B}(J+1, J-1) t+\phi_{B}^{0}(J-1, J+$ 1) with $\Delta E_{B}(J+1, J-1)=B_{B}^{0}[(J+1)(J+2)-(J-1)$ $J]$. Constructive interference between $|B, J+1\rangle \rightarrow|X, J\rangle$ and $|B, J-1\rangle \rightarrow|X, J\rangle$ in emission occurs when $\phi_{B}(J+1$, 
$J-1)(t)=2 N \pi$, while destructive interference occurs when $\phi_{B}(J+1, J-1)(t)=(2 N \pm 1) \pi$, leading to the quantum beat in the time-dependent emission rate with frequency $\Delta E_{B}(J+1, J-1)$.

The difference between the rotational constants $B_{X}^{0}$ and $B_{B}^{0}$ means that there are time windows where destructive interference of the $|X, J\rangle \rightarrow|B, J+1\rangle$ and $|X, J+2\rangle \rightarrow|B, J+1\rangle$ transitions in absorption [when $\phi_{X}(J+2, J)(t)=(2 N \pm 1) \pi$ ] coincides with constructive interference of the $|B, J+1\rangle \rightarrow$ $|X, J\rangle$ and $|B, J-1\rangle \rightarrow|X, J\rangle$ transitions in emission [when $\left.\phi_{B}(J+1, J-1)(t)=2 N \pi\right]$ and vice versa. This leads to the frequency-resolved gain (red) and loss (blue) windows as a function of the pump-probe time delay in Fig. 2(a).

As many four-level systems such as the one shown in Fig. 2(c) are formed by the pump, they generate a rich gain-loss pattern of frequency-resolved transient absorption of the probe pulse [Fig. 2(a)]. Figure 2(a) incorporates the comprehensive modeling of the whole process, starting with the thermal ensemble of neutral molecules and accounting for (i) alignment of the neutral molecule; (ii) its angle-dependent strong-field ionization into the laser-dressed ionic states, with the ratio of $B$ to $X$ state populations at $20 \%$ upon ionization; (iii) entanglement between the photoelectron and the ion, which negates the $X-B$ coherence once the photoelectron is integrated out; (iv) coupled electronic, vibrational, and rotational dynamics in the ion; and (v) comprehensive calculations of transient absorption and emission of the probe pulse, including all interferences. Note that gain windows emerge with neither electronic nor rotational population inversion [cf. Fig. 2(b)].

Figure 2(d) connects the frequency-domain picture of Fig. 2(a) to the time-domain picture of Figs. 1(b) and 1(c). It shows the frequency-integrated absorption from the $X$ state (blue line) and emission (negative absorption) from the $B$ state (red line) stimulated by the short probe pulse as a function of the pump-probe delay. The pattern of each line follows the alignment measure of the respective ion in Fig. 1(b). This is not a coincidence. The same phase differences that control the interferences in transient absorption by each $(|X, J\rangle,|X, J+2\rangle)$ pair control their contribution to the ensemble-averaged $\left\langle\cos ^{2} \theta\right\rangle_{X}$ measure of the field-free rotations in the $X$ state. The same applies to emission by each $(|B, J-1\rangle,|B, J+1\rangle)$ pair and the ensemble-averaged $\left\langle\cos ^{2} \theta\right\rangle_{B}$ measure. As shown in Supplement 1, integrating the frequency-resolved absorption over all frequencies yields the total transient absorption probability $W_{\text {abs }}(\tau) \propto P_{X}\left\langle\cos ^{2}(\theta)\right\rangle_{X}(\tau)$. Analogous results hold for transient emission.

The green line in Fig. 2(d) shows the overall gain-loss balance, integrated over all energies. As expected, the strongest gain windows coincide with anti-alignment of the $X$ state [cf. Figs. 1(b) and $1(\mathrm{c})]$ when the gain-loss pattern in Fig. 2(a) shows gain across the whole spectrum. If the $X$ and $B$ state molecules would antialign simultaneously during their field-free evolution, the net gain would vanish.

The results presented in Fig. 2 are robust with respect to the pump parameters, and the lasing mechanism described above is completely general. The only necessary ingredients are nonnegligible population in the $B$ state and molecular rotations, both unavoidable during the interaction with an intense laser pulse. For the pump pulse parameters and temperature used for Fig. 1 and (a)

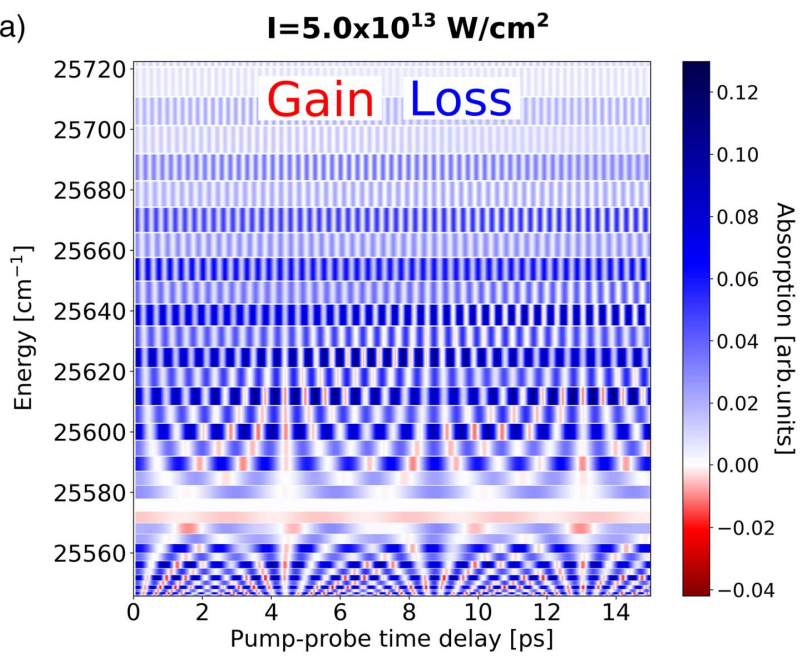

(b)

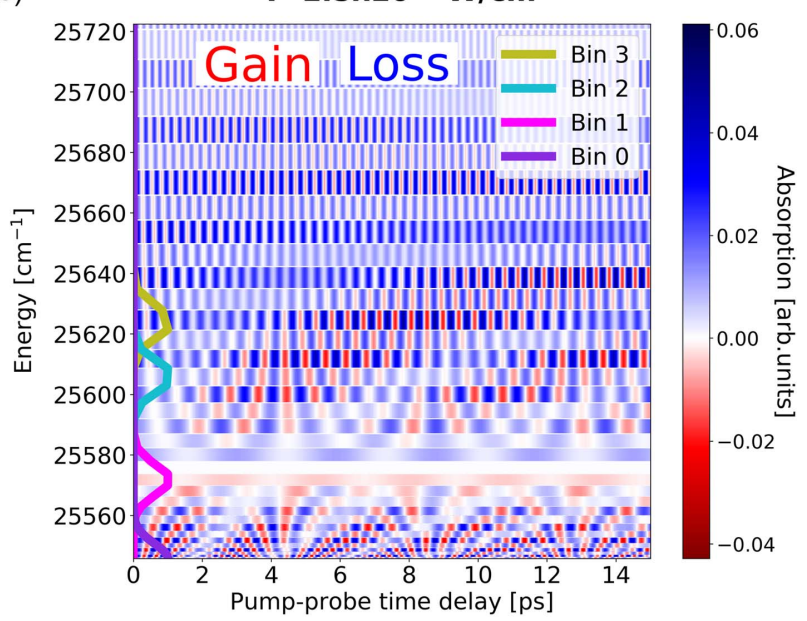

(c)

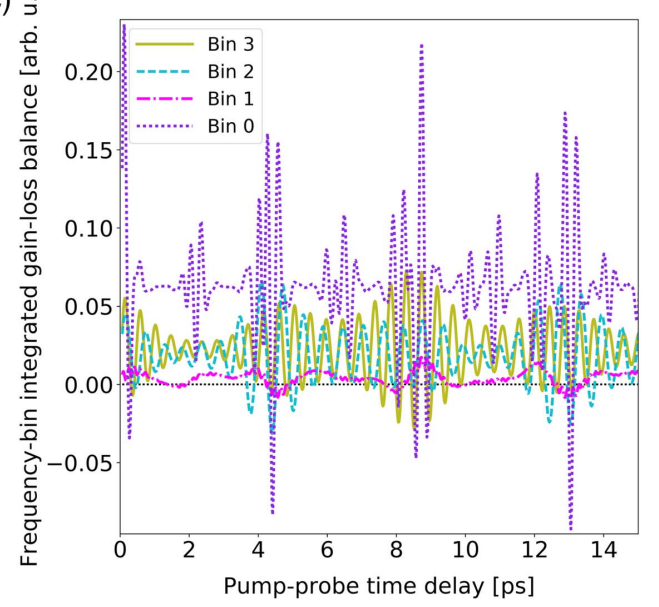

Fig. 3. Rotational quantum beat lasing in $\mathrm{N}_{2}^{+}$for different intensities realistic in femtosecond laser filamentation experiments. Calculations are performed for a $23 \mathrm{fs}, 800 \mathrm{~nm}$ pump pulse and the same probe pulse and temperature as in Fig. 2. (a) and (b) Same as Fig. 2(a), but for pump intensities of (a) $I=5.0 \times 10^{13} \mathrm{~W} / \mathrm{cm}^{2}$ and (b) $I=1.5 \times 10^{14} \mathrm{~W} / \mathrm{cm}^{2}$. (c) Gain-loss balance for $I=1.5 \times 10^{14} \mathrm{~W} / \mathrm{cm}^{2}$ integrated over the frequency windows (bins) indicated in panel (b). For both intensities, gain windows also emerge for the total gain-loss balance integrated over all frequencies, analogue to Fig. 2(d). Gain windows emerge without population inversion of the medium $\left(P_{X}>P_{B}\right)$. 
Fig. 2, our simulations show that net gain sets in already at the ratio $P_{B} / P_{X} \simeq 10 \%$ upon ionization.

Figure 3 shows results for two other pump intensities realistic in laser filamentation (see Supplement 1 for more information on the dependence of gain on the pump intensity). The expected clamping intensity is $I \lesssim 1 \times 10^{14} \mathrm{~W} / \mathrm{cm}^{2}$, but pulse compression during filamentation suggests that short intensity spikes can reach $I \sim 2 \times 10^{14} \mathrm{~W} / \mathrm{cm}^{2}[54,55]$. For $800 \mathrm{~nm}$ driver fields with intensities $I \lesssim 2 \times 10^{14} \mathrm{~W} / \mathrm{cm}^{2}$, the $B$ state population is not strongly affected by laser-driven excitations from $X$. However, the higher the intensity, the stronger the depletion of the $X$ state into $A[25,26]$ (especially for pump pulses shorter or comparable to the $\sim 18 \mathrm{fs}$ vibrational period in the $A$ state), yielding an overall change of the gain-loss balance in favor of gain and hence the emergence of additional gain windows in time; see Figs. 3(b) and $3(\mathrm{c})$.

The results presented in Fig. 3 are in remarkably good agreement with recent experimental findings [38,39]. Ref. [38] reports on pump-probe femtosecond filamentation experiments in a nitrogen gas cell, measuring the delay-dependent amplification of the probe around the $391 \mathrm{~nm}$ line using a high-resolution spectrometer. Our simulations closely reproduce all the main features of the measured time- and frequency-resolved gain, including the parabolic structure of the gain, the fast temporal modulation of each rotational line that increases with $J$ in the $\mathrm{R}$ branch, and the slower temporal modulation, reflecting the molecular alignment dynamics and giving rise to the distinct gain windows, e.g., at $\tau \sim 4.4$ ps, where amplification occurs across the whole spectrum. Experiments that do not resolve each individual rotational line observe (the weighted sum of) frequency-window-integrated, highly oscillating gain-loss structures such as the ones shown in Fig. 3(c). For increasing pump intensity, the oscillating gain starts to occur for virtually all pump-probe time delays as observed in the nitrogen gas jet experiments in Ref. [39].

Taking into account the effects of gain narrowing and inelastic collisions that destroy the rotational coherences in the ionic states, our results reproduce the typical observations made in pumpprobe "air lasing" experiments (see e.g., Refs. [14,21,38,39]): a rapid increase of the gain as a function of the pump-probe delay during the first few hundreds of femtoseconds, followed by a slow decay of the gain for delays up to tens of picoseconds that is periodically modulated by the molecular alignment dynamics.

In the present mechanism, amplification is enabled due to the stimulated emission triggered by the probe, while absorption is suppressed. Thus, the amplified emission can emerge without any delay after the probe, in contrast to the experimentally observed delays of $\sim 1$ to 8 ps between the probe pulse and the enhanced $391 \mathrm{~nm}$ emission reported in Refs. [14,22,24,31,35,37]. This retardation has been attributed to the effect of probe-triggered superradiance [22,24,31,37], whereas the origin of the optical amplification is still debated. Ref. [35] offered an intriguing explanation, evoking long-lived coherences and coupling between the electronic states $X, A$, and $B$, supported by the picosecond-long tail of the pump pulse. Such long tails are explicitly absent in our analysis, which assumes that the pump pulse is fully over within a few tens of femtoseconds, with no temporal overlap between the probe and the pump pulses.

\section{CONCLUSIONS AND OUTLOOK}

We have discussed how molecular ionization and alignment, naturally induced by intense femtosecond laser pulses propagating through molecular gas, can lead to amplification of light in an inversionless medium. Developing a comprehensive theoretical model for studying the amplification effect around the $391 \mathrm{~nm}$ line in $\mathrm{N}_{2}^{+}$in the intensity regime relevant for laser filamentation experiments $\left(I \sim 1-2 \times 10^{14} \mathrm{~W} / \mathrm{cm}^{2}\right)$, we have shown that neither population inversion nor coherences between the electronic states of the ion are required for the air lasing phenomenonthe rotational quantum beat lasing without inversion makes it possible.

In the present mechanism, the gain window travels with the group velocity of the pump pulse so that amplification occurs only in the forward direction. To achieve lasing in the backward direction, highly desirable for remote sensing, one would greatly benefit from creating population inversion, at least between the rotational states. To this end, a sequence of two well-timed pump pulses can be used to control rotations of the $X$ and $B$ states of the ion. Their different rotational periods mean that the second pulse can be timed to simultaneously accelerate rotations of the $B$ state and slow down the rotations of the $X$ state, generating rotational inversion. Optimization of such pulse sequence, including their carrier frequencies, energies, and time delay, should open a route for achieving robust and strong backward lasing in open air.

Funding. Deutsche Forschungsgemeinschaft (IV 152/61, SM 292/5-1); Engineering and Physical Sciences Research Council / Defence Science and Technology Laboratory (MURI EP/N018680/1); Horizon 2020 Framework Programme (641789); Agence Nationale de la Recherche (ANR-10-LABX-0039-PALM).

Acknowledgment. The authors thank Daniil Kartashov, Andrius Baltuška, Mathew Britton, and Paul Corkum for many fruitful and stimulating discussions.

Disclosures. The authors declare no conflicts of interest.

See Supplement 1 for supporting content.

${ }^{\dagger}$ These authors contributed equally to this work.

\section{REFERENCES}

1. O. A. Kocharovskaya and Y. I. Khanin, "Coherent amplification of an ultrashort pulse in a three-level medium without a population inversion," Pis'ma Zh. Eksp. Teor. Fiz. 48, 581 (1988).

2. Y. I. Khanin and O. A. Kocharovskaya, "Inversionless amplification of ultrashort pulses and coherent population trapping in a three-level medium," J. Opt. Soc. Am. B 7, 2016-2024 (1990).

3. M. O. Scully, S.-Y. Zhu, and A. Gavrielides, "Degenerate quantum-beat laser: lasing without inversion and inversion without lasing," Phys. Rev. Lett. 62, 2813-2816 (1989).

4. S. E. Harris, "Lasers without inversion: interference of lifetimebroadened resonances," Phys. Rev. Lett. 62, 1033-1036 (1989).

5. A. Nottelmann, C. Peters, and W. Lange, "Inversionless amplification of picosecond pulses due to Zeeman coherence," Phys. Rev. Lett. 70, 1783-1786 (1993).

6. E. S. Fry, X. Li, D. Nikonov, G. G. Padmabandu, M. O. Scully, A. V. Smith, F. K. Tittel, C. Wang, S. R. Wilkinson, and S.-Y. Zhu, "Atomic coherence effects within the sodium $d_{1}$ line: lasing without inversion via population trapping," Phys. Rev. Lett. 70, 3235-3238 (1993).

7. W. E. van der Veer, R. J. J. van Diest, A. Dönszelmann, and H. B. van Linden van den Heuvell, "Experimental demonstration of light amplification without population inversion," Phys. Rev. Lett. 70, 3243-3246 (1993).

8. A. S. Zibrov, M. D. Lukin, D. E. Nikonov, L. Hollberg, M. O. Scully, V. L. Velichansky, and H. G. Robinson, "Experimental demonstration of laser 
oscillation without population inversion via quantum interference in $\mathrm{Rb}$," Phys. Rev. Lett. 75, 1499-1502 (1995).

9. O. Kocharovskaya, "Amplification and lasing without inversion," Phys. Rep. 219, 175-190 (1992).

10. M. O. Scully, "From lasers and masers to phaseonium and phasers," Phys. Rep. 219, 191-201 (1992).

11. M. O. Scully and M. Fleischhauer, "Lasers without inversion," Science 263, 337-338 (1994)

12. Q. Luo, W. Liu, and S. Chin, "Lasing action in air induced by ultra-fast laser filamentation,” Appl. Phys. B 76, 337-340 (2003).

13. J. Yao, B. Zeng, H. Xu, G. Li, W. Chu, J. Ni, H. Zhang, S. L. Chin, Y Cheng, and Z. Xu, "High-brightness switchable multiwavelength remote laser in air," Phys. Rev. A 84, 051802 (2011).

14. J. Yao, G. Li, C. Jing, B. Zeng, W. Chu, J. Ni, H. Zhang, H. Xie, C. Zhang H. Li, H. Xu, S. L. Chin, Y. Cheng, and Z. Xu, "Remote creation of coherent emissions in air with two-color ultrafast laser pulses," New J. Phys. 15, 023046 (2013).

15. Y. Liu, Y. Brelet, G. Point, A. Houard, and A. Mysyrowicz, "Self-seeded lasing in ionized air pumped by $800 \mathrm{~nm}$ femtosecond laser pulses," Opt. Express 21, 22791-22798 (2013).

16. W. Chu, G. Li, H. Xie, J. Ni, J. Yao, B. Zeng, H. Zhang, C. Jing, H. Xu, Y. Cheng, and Z. Xu, "A self-induced white light seeding laser in a femtosecond laser filament," Laser Phys. Lett. 11, 015301 (2013).

17. A. Braun, G. Korn, X. Liu, D. Du, J. Squier, and G. Mourou, "Selfchanneling of high-peak-power femtosecond laser pulses in air," Opt. Lett. 20, 73-75 (1995).

18. A. Couairon and A. Mysyrowicz, "Femtosecond filamentation in transparent media," Phys. Rep. 441, 47-189 (2007).

19. L. Bergé, S. Skupin, R. Nuter, J. Kasparian, and J.-P. Wolf, "Ultrashort filaments of light in weakly ionized, optically transparent media," Rep. Prog. Phys. 70, 1633-1713 (2007)

20. J. Ni, W. Chu, C. Jing, H. Zhang, B. Zeng, J. Yao, G. Li, H. Xie, C. Zhang H. Xu, S.-L. Chin, Y. Cheng, and Z. Xu, "Identification of the physical mechanism of generation of coherent emissions in air by femtosecond laser excitation," Opt. Express 21, 8746-8752 (2013).

21. H. Zhang, C. Jing, J. Yao, G. Li, B. Zeng, W. Chu, J. Ni, H. Xie, H. Xu, S. L. Chin, K. Yamanouchi, Y. Cheng, and Z. Xu, "Rotational coherence encoded in an "air-laser" spectrum of nitrogen molecular ions in an intense laser field," Phys. Rev. X 3, 041009 (2013).

22. G. Li, C. Jing, B. Zeng, H. Xie, J. Yao, W. Chu, J. Ni, H. Zhang, H. Xu, Y. Cheng, and Z. Xu, "Signature of superradiance from a nitrogen-gas plasma channel produced by strong-field ionization," Phys. Rev. A 89, 033833 (2014).

23. S. Mitryukovskiy, Y. Liu, P. Ding, A. Houard, A. Couairon, and A. Mysyrowicz, "Plasma luminescence from femtosecond filaments in air: evidence for impact excitation with circularly polarized light pulses," Phys. Rev. Lett. 114, 063003 (2015).

24. Y. Liu, P. Ding, G. Lambert, A. Houard, V. Tikhonchuk, and A Mysyrowicz, "Recollision-induced superradiance of ionized nitrogen molecules," Phys. Rev. Lett. 115, 133203 (2015).

25. H. Xu, E. Lötstedt, A. Iwasaki, and K. Yamanouchi, "Sub-10-fs population inversion in $\mathrm{N}_{2}{ }^{+}$air lasing through multiple state coupling," Nat. Commun. 6, 8347 (2015).

26. J. Yao, S. Jiang, W. Chu, B. Zeng, C. Wu, R. Lu, Z. Li, H. Xie, G. Li, C. Yu, Z. Wang, H. Jiang, Q. Gong, and Y. Cheng, "Population redistribution among multiple electronic states of molecular nitrogen ions in strong laser fields," Phys. Rev. Lett. 116, 143007 (2016).

27. D. Kartashov, S. Haessler, S. Ališauskas, G. Andriukaitis, A. Pugžlys, A. Baltuška, J. Möhring, D. Starukhin, M. Motzkus, A. Zheltikov, M. Richter, F. Morales, O. Smirnova, M. Y. Ivanov, and M. Spanner, "Transient inversion in rotationally aligned nitrogen ions in a femtosecond filament," in Research in Optical Sciences, OSA Technical Digest (online) (Optical Society of America, 2014), paper HTh4B.5.

28. M. Richter, F. Morales, M. Spanner, O. Smirnova, and M. Ivanov, "Optical lasing during laser filamentation in the nitrogen molecular ion: Ro-vibrational inversion," in 2017 Conference on Lasers and Electro-Optics Europe \& European Quantum Electronics Conference (CLEO/Europe-EQEC), Munich (2017).

29. A. Azarm, P. Corkum, and P. Polynkin, "Optical gain in rotationally excited nitrogen molecular ions," Phys. Rev. A 96, 051401 (2017).

30. M. Lei, C. Wu, A. Zhang, Q. Gong, and H. Jiang, "Population inversion in the rotational levels of the superradiant $\mathrm{N}_{2}{ }^{+}$pumped by femtosecond laser pulses," Opt. Express 25, 4535-4541 (2017).

31. X. Zhong, Z. Miao, L. Zhang, Q. Liang, M. Lei, H. Jiang, Y. Liu, Q. Gong, and $\mathrm{C}$. Wu, "Vibrational and electronic excitation of ionized nitrogen molecules in intense laser fields," Phys. Rev. A 96, 043422 (2017).

32. H. Xu, E. Lötstedt, T. Ando, A. Iwasaki, and K. Yamanouchi, "Alignmentdependent population inversion in $\mathrm{N}_{2}^{+}$in intense few-cycle laser fields," Phys. Rev. A 96, 041401 (2017).
33. Y. Liu, P. Ding, N. Ibrakovic, S. Bengtsson, S. Chen, R. Danylo, E. R. Simpson, E. W. Larsen, X. Zhang, Z. Fan, A. Houard, J. Mauritsson, A. L'Huillier, C. L. Arnold, S. Zhuang, V. Tikhonchuk, and A. Mysyrowicz, "Unexpected sensitivity of nitrogen ions superradiant emission on pump laser wavelength and duration," Phys. Rev. Lett. 119, 203205 (2017).

34. M. Britton, P. Laferrière, D. H. Ko, Z. Li, F. Kong, G. Brown, A. Naumov, C Zhang, L. Arissian, and P. B. Corkum, "Testing the role of recollision in $\mathrm{N}_{2}^{+}$ air lasing," Phys. Rev. Lett. 120, 133208 (2018).

35. A. Mysyrowicz, R. Danylo, A. Houard, V. Tikhonchuk, X. Zhang, Z. Fan, Q. Liang, S. Zhuang, L. Yuan, and Y. Liu, "Lasing without population inversion in $\mathrm{N}_{2}^{+}$," APL Photon. 4, 110807 (2019).

36. B. Xu, S. Jiang, J. Yao, J. Chen, Z. Liu, W. Chu, Y. Wan, F. Zhang, L. Qiao, R. Lu, Y. Cheng, and Z. Xu, "Free-space $\mathrm{N}_{2}^{+}$lasers generated strong laser fields: the role of molecular vibration," Opt. Express 26, 13331-13339 (2018).

37. X. Zhong, Z. Miao, L. Zhang, H. Jiang, Y. Liu, Q. Gong, and C. Wu, "Optimizing the 391-nm lasing intensity from ionized nitrogen molecules in 800-nm femtosecond laser fields," Phys. Rev. A 97, 033409 (2018).

38. L. Arissian, B. Kamer, A. Rastegari, D. M. Villeneuve, and J.-C. Diels, "Transient gain from $\mathrm{N}_{2}^{+}$in light filaments," Phys. Rev. A 98, 053438 (2018).

39. M. Britton, M. Lytova, P. Laferrière, P. Peng, F. Morales, D. H. Ko, M. Richter, P. Polynkin, D. M. Villeneuve, C. Zhang, M. Ivanov, M. Spanner, L. Arissian, and P. B. Corkum, "Short- and long-term gain dynamics in $\mathrm{N}_{2}{ }^{+}$air lasing," Phys. Rev. A 100, 013406 (2019).

40. H. Li, Q. Song, J. Yao, Z. Liu, J. Chen, B. Xu, K. Lin, J. Qiang, B. He, H. Xu, Y. Cheng, H. Zeng, and J. Wu, "Air lasing from singly ionized $\mathrm{N}_{2}$ driven by bicircular two-color fields," Phys. Rev. A 99, 053413 (2019).

41. T. Ando, E. Lötstedt, A. Iwasaki, H. Li, Y. Fu, S. Wang, H. Xu, and K. Yamanouchi, "Rotational, vibrational, and electronic modulations in $\mathrm{N}_{2}^{+}$ lasing at $391 \mathrm{~nm}$ : evidence of coherent $\mathrm{B}^{2} \Sigma_{u}^{+}-\mathrm{X}^{2} \Sigma_{g}^{+}-\mathrm{A}^{2} \Pi_{u}$ coupling," Phys. Rev. Lett. 123, 203201 (2019).

42. H. Li, M. Hou, H. Zang, Y. Fu, E. Lötstedt, T. Ando, A. Iwasaki, K Yamanouchi, and $\mathrm{H}$. Xu, "Significant enhancement of $\mathrm{N}_{2}{ }^{+}$lasing by polarization-modulated ultrashort laser pulses," Phys. Rev. Lett. 122 013202 (2019).

43. A. Zhang, Q. Liang, M. Lei, L. Yuan, Y. Liu, Z. Fan, X. Zhang, S. Zhuang C. Wu, Q. Gong, and H. Jiang, "Coherent modulation of superradiance from nitrogen ions pumped with femtosecond pulses," Opt. Express 27, 12638-12646 (2019)

44. A. Zhang, M. Lei, J. Gao, C. Wu, Q. Gong, and H. Jiang "Subfemtosecond-resolved modulation of superfluorescence from ionized nitrogen molecules by $800-n m$ femtosecond laser pulses," Opt. Express 27, 14922-14930 (2019).

45. Q. Zhang, H. Xie, G. Li, X. Wang, H. Lei, J. Zhao, Z. Chen, J. Yao, Y Cheng, and Z. Zhao, "Sub-cycle coherent control of ionic dynamics via transient ionization injection," Communications Phys. 3, 50 (2020).

46. J. Kasparian, R. Sauerbrey, and S. L. Chin, "The critical laser intensity of self-guided light filaments in air," Appl. Phys. B 71, 877-879 (2000).

47. S. Xu, J. Bernhardt, M. Sharifi, W. Liu, and S. L. Chin, "Intensity clamping during laser filamentation by TW level femtosecond laser in air and argon," Laser Phys. 22, 195-202 (2012).

48. P. M. Solyankin, I. A. Nikolaeva, A. A. Angeluts, D. E. Shipilo, N. V. Minaev, N. A. Panov, A. V. Balakin, Y. Zhu, O. G. Kosareva, and A. P. Shkurinov, "THz generation from laser-induced breakdown in pressurized molecular gases: on the way to terahertz remote sensing of the atmospheres of Mars and Venus," New J. Phys. 22, 013039 (2020).

49. T. Seideman, "Revival structure of aligned rotational wave packets," Phys. Rev. Lett. 83, 4971-4974 (1999).

50. P. W. Dooley, I. V. Litvinyuk, K. F. Lee, D. M. Rayner, M. Spanner, D. M. Villeneuve, and P. B. Corkum, "Direct imaging of rotational wave-packet dynamics of diatomic molecules," Phys. Rev. A 68, 023406 (2003).

51. A. K. Popov and V. V. Slabko, "Inversionless amplification by anisotropic molecules," Opt. Lett. 30, 1719-1721 (2005).

52. M. Spanner and S. Patchkovskii, "One-electron ionization of multielectron systems in strong nonresonant laser fields," Phys. Rev. A 80 , 063411 (2009).

53. Y. Zhang, E. Lötstedt, and K. Yamanouchi, "Mechanism of population inversion in laser-driven $\mathrm{N}_{2}^{+}$," J. Phys. B 52, 055401 (2019).

54. M. B. Gaarde and A. Couairon, "Intensity spikes in laser filamentation: diagnostics and application," Phys. Rev. Lett. 103, 043901 (2009).

55. S. I. Mitryukovskiy, Y. Liu, A. Houard, and A. Mysyrowicz, "Re-evaluation of the peak intensity inside a femtosecond laser filament in air," J. Phys. B 48, 094003 (2015). 